\title{
The Latest Advances in Imaging Crosstalk Between the Immune System and Fibrosis in Cardiovascular Disease
}

\author{
Gyu Seong Heo*, Lanlan Lou*, Deborah Sultan, and Yongjian Liu \\ Mallinckrodt Institute of Radiology, Washington University, St. Louis, Missouri
}

\begin{abstract}
Inflammation and fibrosis are hallmarks of tissue repair processes and organ failure progression in cardiovascular diseases. Paradigm-shifting research on diverse immune cell populations within the cardiovascular system have enabled discovery of new biomarkers fostering development of diagnostic and therapeutic agents at the molecular level to better manage cardiovascular diseases. To date, a variety of molecular imaging agents have been developed to visualize the biomarkers expressed on immune cells and fibroblasts within their crosstalk network, which drives the pathogenesis of fibrosis triggered by both innate and adaptive immunity. Herein, key biomarkers upregulated in the immune-fibrosis axis are discussed. The promising molecular imaging agents to reveal this critical pathologic process are summarized.
\end{abstract}

Key Words: immune system; inflammation; fibrosis; cardiovascular diseases; molecular imaging

J Nucl Med 2021; 62:1341-1346

DOI: 10.2967/jnumed.120.255539

F ibrosis, a scarring process, is defined as an uncontrolled accumulation of extracellular matrix (ECM) molecules on injured tissues and ultimately leads to adverse tissue remodeling, organ damage, and failure. Inflammatory and immunologic reactions involving both innate and adaptive immune systems are the underlying players driving fibrosis $(1,2)$. In cardiovascular diseases (CVDs), vascular remodeling triggered by inflammatory stimuli is significantly associated with atherogenesis, deposition of ECM proteins on the arterial wall, and eventually vascular fibrosis. It is known that cardiac fibrosis is a major contributor to many CVDs, including myocardial infarction (MI) (3). Clinical diagnostics mostly provide an anatomic characterization of fibrotic scars at the stage when disease is irreversible and irreparable. The limited options of antifibrotic medications in the clinic compel an urgent search for novel diagnostic methods using molecular imaging to identify new biomarkers overexpressed during immune response and tissue repair within the immune-fibrosis network at an early stage for potential intervention and theranostics $(4,5)$.

Many molecular probes have been developed for inflammation and fibrosis imaging in CVDs $(6,7)$. However, the mechanism of crosstalk between immune cells and fibroblasts is not fully understood. Detection of the early onset of immune response and

Received December 1, 2020; revision accepted April 13, 2021.

For correspondence or reprints, contact Yongjian Liu (yongjianliu@wustl.edu). ${ }^{*}$ Contributed equally to this work.

Published online April 16, 2021.

COPYRIGHT (C) 2021 by the Society of Nuclear Medicine and Molecular Imaging. wound healing process, such as activation of immune cells and subsequent fibrotic response, is underexplored. Herein, we briefly discuss current imaging research on targets upregulated in the molecular and cellular pathways of the immune-fibrosis crosstalk network. We focus on PET and SPECT radiotracers because of their high sensitivity, quantitative measurement, and well-established translational strategies $(4,8,9)$.

\section{IMMUNE CELLS INVOLVED IN INFLAMMATION, TISSUE REPAIR, AND FIBROGENESIS}

CVDs arise from various types of injurious stimuli on heart or blood vessels, either acute (e.g., ischemia/reperfusion [I/R] injury in MI) or chronic (e.g., cholesterol deposition for atherosclerosis). After injury, the immune system is activated and initiates a wound healing process to minimize damage and restore function to injured tissues (Fig. 1) (1-3,10-12). Within minutes of injury, damaged, stressed, and dying cells release damage-associated molecular patterns, which bind to pattern recognition receptors, including toll-like receptors and receptors for advanced glycation end products, which are expressed on surviving adjacent cells and leukocytes. Stimulation of these pattern recognition receptors activates complementary signaling pathways for not only proinflammatory cytokines and chemokines but also cell adhesion molecules. These inflammatory mediators promote the recruitment of leukocytes, including neutrophils and proinflammatory monocytes expressing high levels of Ly6C (Ly6 $\mathrm{C}^{\text {high }}$ monocytes in mice), to remove damaged cells by efferocytosis and release enzymes (proteases and oxidases) for tissue digestion. After the clearance of neutrophils, monocytes expressing low levels of Ly6C (Ly6Clow monocytes in mice) are recruited to the lesion and differentiate into reparative macrophages, which secrete antiinflammatory mediators such as transforming growth factor (TGF)- $\beta$ and interleukin-10 to promote myofibroblast and vascular cell infiltration for tissue repair and regeneration. Macrophages interact with fibroblasts via secreting cytokines, chemokines, and other factors such as high levels of MMPs, which cause extensive matrix breakdown, altering the mechanical properties of the tissues to increase the expression of tumor necrosis factor- $\alpha$, TGF- $\alpha$, and TGF- $\beta$ (13). Additionally, the loss of interleukin- $1 \beta$ and interleukin-10 expression during the proliferative phase allows fibroblasts to transdifferentiate into myofibroblasts, which produce ECM proteins to help maintain the structural integrity of injured tissues (14). During this dynamic and phasic process, crosstalk between the immune system and fibrosis plays a crucial role in regulating the secretion of proinflammatory and antiinflammatory mediators, fibrogenesis, remodeling, and tissue repair. Therefore, the real-time detection of biomarkers overexpressed by immune cells and fibroblasts and targets elevated during their interactions may facilitate comprehension of the 


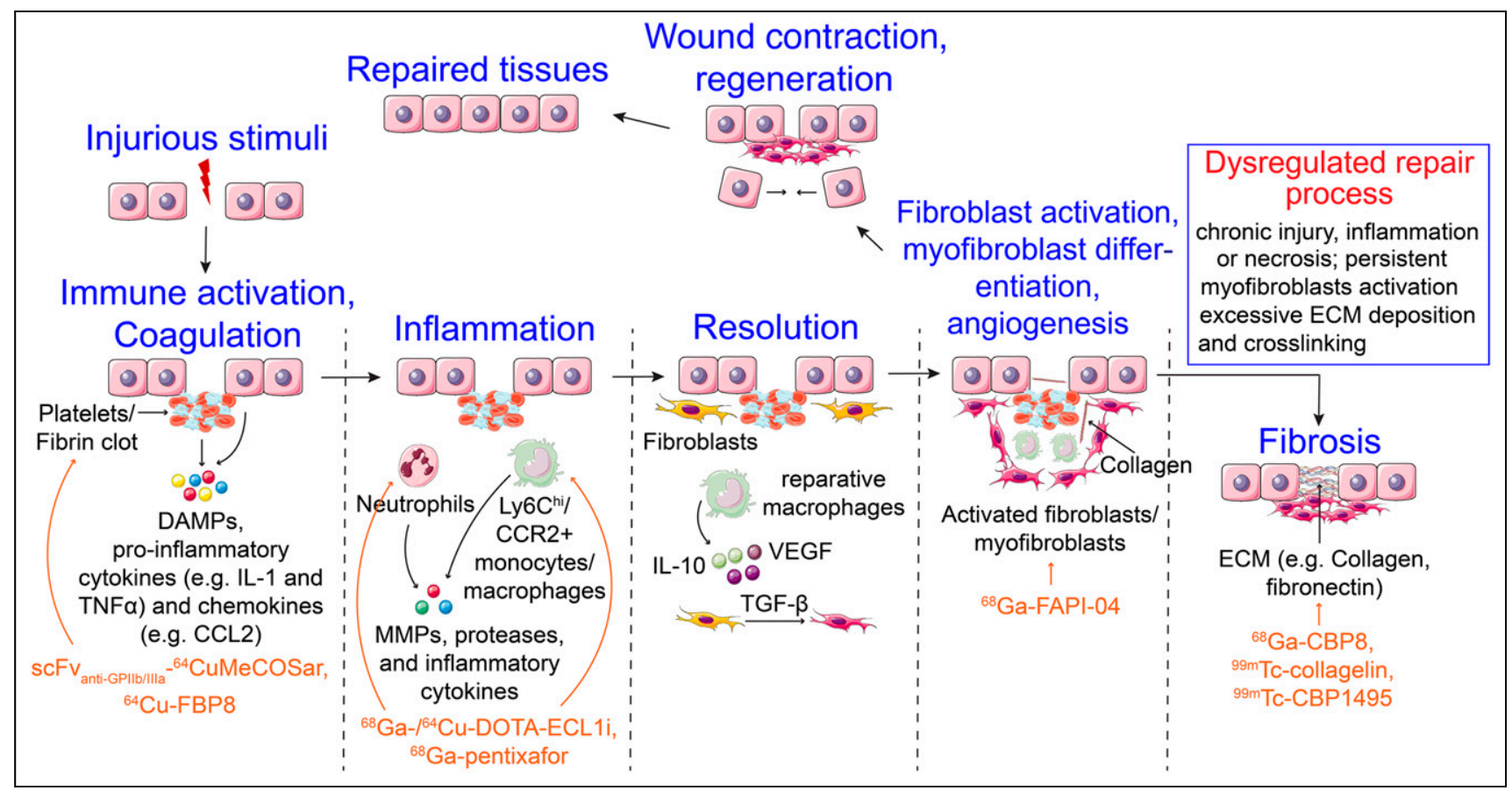

FIGURE 1. Molecular and cellular processes of immune cells involved in inflammation, tissue repair, and fibrosis. (Adapted with permission of (4).) DAMPs = damage-associated molecular patterns; IL-10 = interleukin 10; TNF = tumor necrosis factor; VEGF = vascular endothelial growth factor.

underlying mechanism of the crosstalk and illuminate the discovery of targeted treatment for timely intervention to improve patient outcome (3).

\section{MOLECULAR IMAGING OF CROSSTALK BETWEEN IMMUNE SYSTEM AND FIBROSIS IN CVDS}

\section{Immune Cell Imaging}

Because of the elevated expression of CXCR4 on multiple leukocytes after cardiovascular/cardiac injury, much effort has been devoted to the development of CXCR4-targeting radiotracers. At day 3 after $\mathrm{I} / \mathrm{R}$ injury in mice, ${ }^{68} \mathrm{Ga}$-pentixafor uptake was determined at the site of infarct, with signal proportional to leukocyte infiltration (6). In humans, ${ }^{68} \mathrm{Ga}$-pentixafor demonstrated heterogeneous PET signals in hearts between days 4 and 6 after MI, suggesting alternative regulation of chemokine signaling and inflammatory response (15). Through the combination with plerixafor for targeted intervention, improved treatment efficacy was observed in MI mice when treatment was administrated at high ${ }^{68}$ Ga-pentixafor uptake compared with those at low PET signals. This was illustrated with improved left ventricular (LV) remodeling and cardiac function measured at $6 \mathrm{wk}$ after MI, as well as fewer neutrophils and Ly6 $\mathrm{C}^{\text {high }}$ monocytes in LV (16), which highlighted the importance of CXCR4 PET measuring the spatiotemporal distribution of CXCR4-positive (+) cells to optimize the treatment outcome.

Monocytes and macrophages are indispensable effector cells involved in tissue repair and remodeling. The remarkable heterogeneity of macrophage populations in CVDs is well documented and encompasses their distinct functions in promotion of inflammation, tissue repair and regeneration, and inflammation resolution (1). Because of the dynamic variation of macrophage lineage populations, spatiotemporal detection of macrophage subtypes could facilitate the understanding of their identities, origins, and functions along the initiation and progression of the inflammation-fibrosis axis.

After MI in mice, the composition and ontogeny of macrophages are dramatically shifted. Ly6 $\mathrm{C}^{\text {high }}, \mathrm{CCR} 2+$ monocytes infiltrate the heart, replace resident cardiac macrophages (CCR2-negative [-]), and differentiate into CCR2 + macrophages to stimulate proinflammatory responses and collateral tissue damage and ultimately contribute to heart failure pathogenesis (17). In mice with acute autoimmune myocarditis, siRNA silencing of CCR2 significantly decreased the number of Ly6 $\mathrm{C}^{\text {high }}$ monocytes in hearts and led to a reduction of LV fibrosis (18). These findings implicate the role of infiltrating CCR2+ monocytes and macrophages as important mediators of heart failure pathogenesis and the potential of CCR2targeted therapies to improve outcomes of MI patients. In mouse models of sterile cardiac injury, ${ }^{68}$ Ga-DOTA-extracellular loop 1 inverso (ECL1i) specifically detected infiltrating CCR2+ monocytes and macrophages into the injured heart (Fig. 2) with a loss of signal in CCR2 $2^{-/-}$mice. Tracer uptake in the injured myocardium at day 4 showed a linear correlation with LV function and infarct size measured on day 28 after I/R injury, demonstrating its potential predictive value for adverse effects governed by CCR2 + leukocyte subsets (19). Moreover, ${ }^{64} \mathrm{Cu}$-DOTA-ECL1i not only showed comparable imaging efficiency to ${ }^{68}$ Ga-DOTA-ECL1i in mouse heart injury models (20) but also has been used to track CCR2+ monocytes and macrophages in atherosclerosis and other fibrotic diseases $(21,22)$. Ongoing clinical studies will further evaluate the performance of ${ }^{64} \mathrm{Cu}$-DOTA-ECL1i for tracking CCR2 + cells in humans (23). Recently, an ${ }^{18}$ F-radiolabeled small molecule was also developed for CCR2 preclinical imaging (24).

Matrix metalloproteinases (MMPs) are a multigene family of endopeptidases that selectively digest individual components of 


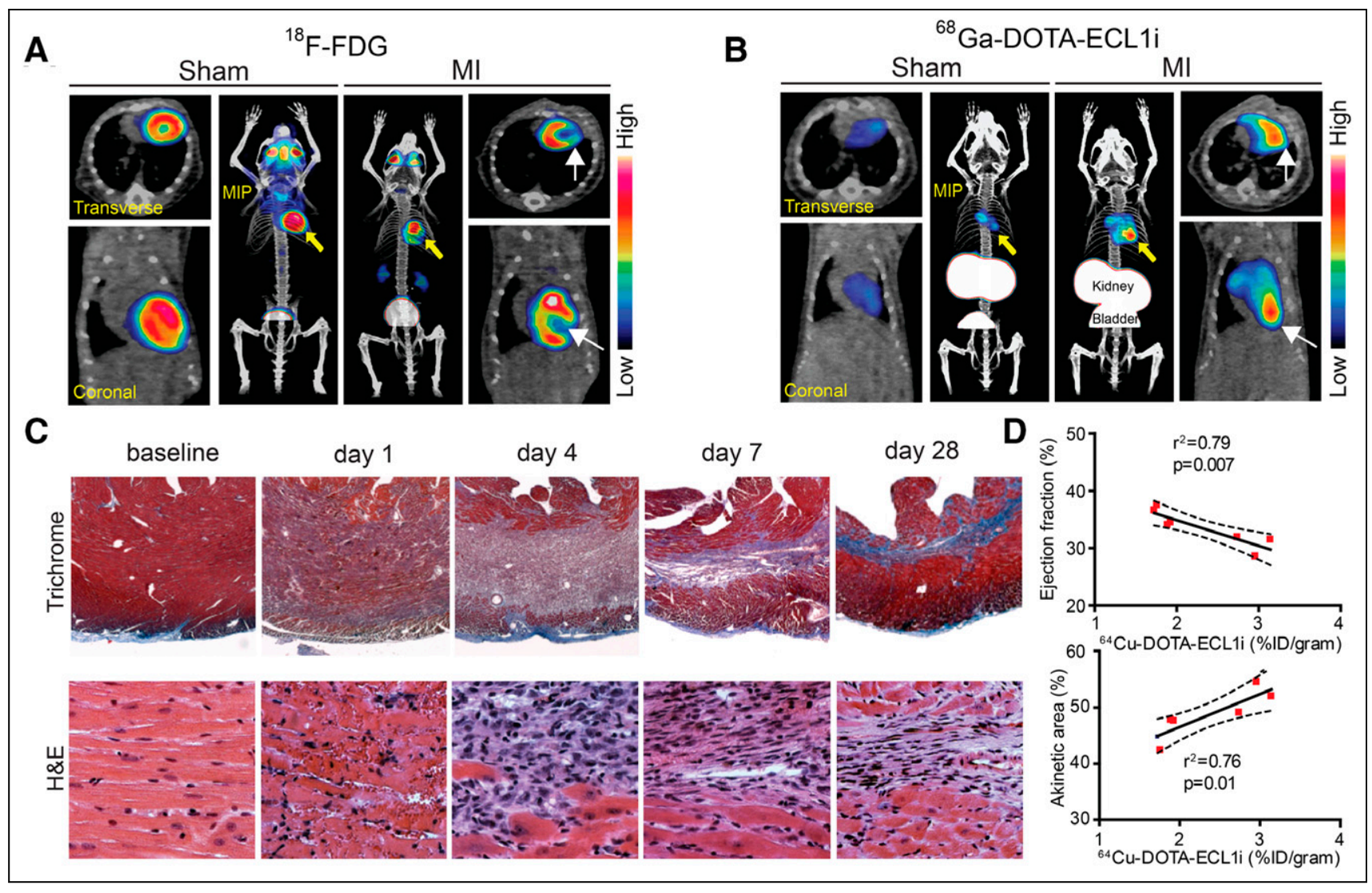

FIGURE 2. PET of ${ }^{68} \mathrm{Ga}$-DOTA-ECL1i in mouse model of closed-chest $\mathrm{l} / \mathrm{R}$ injury. (A) Representative ${ }^{18} \mathrm{~F}-\mathrm{FDG} \mathrm{PET} / \mathrm{CT}$ images obtained $5 \mathrm{~d}$ after $90 \mathrm{~min}$ of $\mathrm{l} /$ $R$ injury identifying infarct region in mice that underwent I/R compared with sham controls. Transverse, coronal, and maximal-intensity-projection views are shown, and white arrows denote infarct area. (B) ${ }^{68}$ Ga-DOTA-ECL1i PET/CT images showing regional accumulation of ${ }^{68}$ Ga-DOTA-ECL1i signal in infarct and border zone $4 \mathrm{~d}$ after $\mathrm{l} / \mathrm{R}$ injury. Yellow arrows identify tracer uptake in hearts that underwent $\mathrm{l} / \mathrm{R}$ injury compared with sham controls. White arrows denote infarct area as determined by ${ }^{18} \mathrm{~F}$-FDG imaging. (C) Trichrome and hematoxylin and eosin (H\&E) staining show evolution of fibrosis (trichrome-blue, $\times 40$ ) and cell infiltration (H\&E, $\times 200$ ) over time in closed-chest I/R injury model. Dense accumulation of cells is seen within infarct $4 \mathrm{~d}$ after $\mathrm{l} / \mathrm{R}$ injury. (D) Linear regression analyses showing relationship between ${ }^{68} \mathrm{Ga}-\mathrm{DOTA}$-ECL1i heart uptake measured on day 4 and echocardiographic assessment of LV ejection fraction and akinetic area measured on day 28 after l/R injury. \%ID = percentage injected dose. (Reprinted with permission of (19).)

ECM. Their activities are associated with tissue remodeling, including recruitment and migration of immune cells and promotion of angiogenesis and apoptosis, making them attractive targets for inflammation and fibrosis imaging (25). An MMP-2 inhibitor, RP805, and a pan-MMP inhibitor, RYM1, were both radiolabeled with ${ }^{99 \mathrm{~m}} \mathrm{Tc}$ for CVD imaging in preclinical models using SPECT $(5,26)$. In contrast to ${ }^{99 \mathrm{~m}} \mathrm{Tc}-\mathrm{RP} 805,{ }^{99 \mathrm{~m}} \mathrm{Tc}-\mathrm{RYM} 1$ had desirable pharmacokinetics and low blood retention. In a mouse abdominal aortic aneurysm model, ${ }^{99 \mathrm{~m}} \mathrm{Tc}-\mathrm{RYM} 1 \mathrm{uptake}$ at aneurysm correlated with CD68 macrophage and activated MMP activity, indicating its potential for inflammation and fibrosis imaging.

Besides CCR2 and MMP, a variety of radiotracers have been developed for macrophages by targeting other chemokine receptors, somatostatin receptors, translocator proteins, and mannose receptors $(6,27)$. Further studies are warranted using these radiotracers to image the subtypes of macrophages, shedding light on their varied roles in the inflammation-fibrosis axis.

\section{Fibroblast and Myofibroblast Imaging}

Fibroblasts not only modulate the recruitment of immune cells but also regulate their behavior, retention, and survival in damaged tissue. Cardiac fibroblasts contribute to myocardial homeostasis by synthesizing and maintaining the ECM network critical for structural and functional integrity. When activated, fibroblasts express cytoplasmic actin and adhesion complexes, permitting migration to the injury site. On differentiation, fibroblasts become a phenotypically distinct cell referred to as a myofibroblast, which is the key cellular effector for tissue repair and fibrogenesis (28). Myofibroblasts produce and deposit structural ECM proteins, including collagen, fibronectin, and elastin, in injured tissues. They release proteases such as MMPs and their inhibitors regulating matrix remodeling. Therefore, activated fibroblasts and myofibroblasts are undisputable target cell populations for molecular imaging to predict outcomes of tissue repair and remodeling process in CVDs.

Fibroblast activation protein (FAP) exhibits a specific expression on activated fibroblasts, making it a promising cell surface biomarker for targeted imaging of fibrotic diseases. Because of its upregulation on cancer-associated fibroblasts, various radiolabeled FAP inhibitors have been developed for tumor imaging (29). In a mouse model of hypertensive cardiac injury and fibrosis, depletion of FAP + fibroblasts reduced myocardial fibrosis and restored cardiac function, indicating the potential of FAP for CVD imaging and therapy $(29,30)$. Through ${ }^{68} \mathrm{Ga}$ radiolabeling of a FAP inhibitor, ${ }^{68}$ Ga-FAPI-04 specifically determined the activated fibroblasts in 


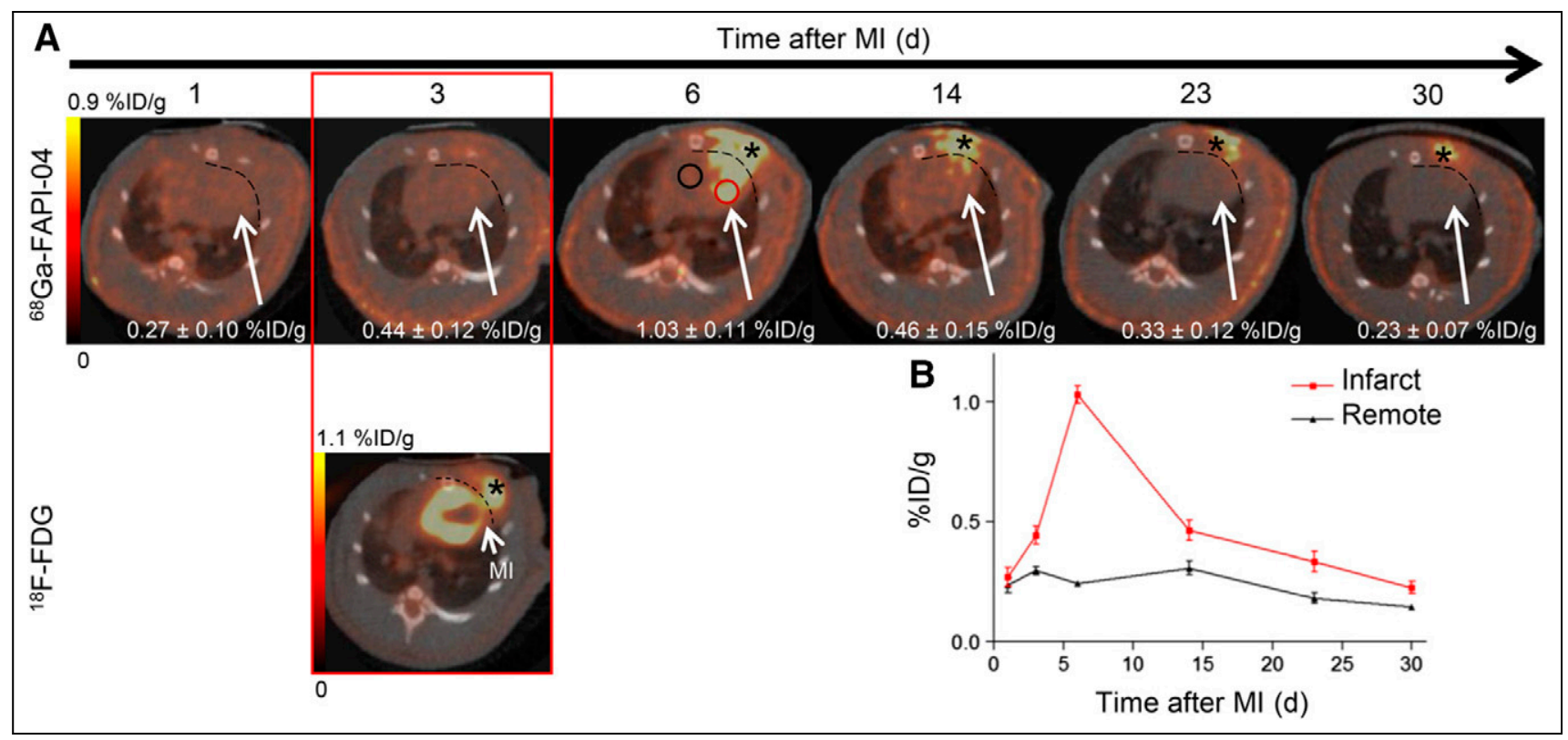

FIGURE 3. ${ }^{68} \mathrm{Ga}-\mathrm{FAPI}-04 \mathrm{PET} / \mathrm{CT}$ in rat MI model. (A) Static PET/CT matched axial slices in same rat subjected to coronary ligation and scanned $1 \mathrm{~h}$ after injection of ${ }^{68} \mathrm{Ga}-\mathrm{FAPI}-04\left(1,3,6,14,23\right.$, and $30 \mathrm{~d}$ after MI) and ${ }^{18} \mathrm{~F}-\mathrm{FDG}$ ( $3 \mathrm{~d}$ after MI). Dashed lines separate tracer uptake in myocardium from uptake in surgical wounds. At day 6 , representative regions of interest drawn over infarct border zone and remote myocardium are illustrated as red and black circles, respectively. (B) Corresponding time-activity curves for infarcted and noninfarcted heart tissue (mean $\pm \mathrm{SD}, n=3) .{ }^{68} \mathrm{Ga}-\mathrm{FAPI}-04$ and ${ }^{18} \mathrm{~F}-\mathrm{FDG}$ exhibited elevated uptake in scars from operation (asterisk). \%ID = percentage injected dose. (Reprinted from (31).)

injured heart in a rat MI model (Fig. 3) (31). In humans, a retrospective analysis of ${ }^{68} \mathrm{Ga}-\mathrm{FAPI}-04$ imaging in cancer patients revealed an association between tracer uptake by the heart and LV ejection fraction, indicating its potential for risk stratification regarding early detection or progression of LV remodeling $(7,32)$. Therefore, molecular imaging of activated fibroblasts and myofibroblasts has great potential for assessing the probability and complications of fibrosis in CVDs, providing information to optimize treatment, and monitoring treatment response for better management.

Because of the pivotal role of angiotensin-converting enzyme inhibitors in the treatment of CVDs, angiotensin-converting enzyme inhibitor-based tracers are of interest for monitoring disease progression and the effectiveness of therapeutic interventions. Many angiotensin-converting enzyme inhibitors, such as ${ }^{18} \mathrm{~F}$ captopril, have been used to image ventricular remodeling after MI in animal models (9). Moreover, the expression of angiotensin II receptor type 1 on fibroblasts and myofibroblasts triggered the radiolabeling of angiotensin II receptor type 1 antagonists such as ${ }^{11} \mathrm{C}-\mathrm{KR} 31173$ for post-MI remodeling and fibrosis imaging (9). Because of the upregulation of $\alpha_{\mathrm{v}} \beta_{3}$ integrin on activated fibroblasts, several radiotracers have been developed $(4,11)$. However, its expression on other cells, including macrophages and endothelial cells, warrants further investigation to ascertain its value for imaging activated fibroblasts.

\section{Activated Platelets}

Besides their role in hemostasis, additional functions of platelets have been uncovered in regeneration and remodeling of injured tissue, including immune cell recruitment, apoptosis, angiogenesis, and ECM formation (33). Activated platelets are involved in immune responses through expression of a variety of membrane receptors (e.g., CD40 ligand) and the release of soluble inflammatory mediators (e.g., TGF- $\beta 1$, CCL5, and CXCL12), which further promote the production of ECM from myofibroblasts. In ST-elevation MI patients, platelet activities were associated with adverse LV remodeling and fibrosis, indicating their potential not only as an imaging biomarker for the early assessment of tissue repair process but also as therapeutic targets (34). Moreover, on activation, the major platelet integrin glycoprotein GPIIb/IIIa $\left(\alpha_{\mathrm{IIb}}\right)$ $\beta_{\text {IIIa }}$; CD41/CD61) undergoes a conformation change, making the altered conformation a unique targeting epitope for the detection of activated platelets. Through ${ }^{64} \mathrm{Cu}$ radiolabeling, the single-chain antibody tracer $\left(\mathrm{scFv}_{\text {anti-GPIIb/IIIa }}{ }^{64} \mathrm{CuMeCOSar}\right)$ revealed significantly higher uptake in the ischemic myocardium compared with the nonischemic region in an $\mathrm{I} / \mathrm{R}$ injury mouse model, suggesting its further evaluation to predict outcomes of subsequent tissue repair processes (35).

\section{Targets Expressed on Thrombus}

Thrombosis is a common pathology underlying ischemic heart disease, ischemic stroke, and venous thromboembolism triggered by either a mechanical injury or the rupture of an atherosclerotic plaque. Molecular imaging of the components involved in thrombus formation may afford accurate and early detection of thrombosis to minimize the risk of complications for improved treatment (36). Blood coagulation factor (FXIII) is an enzyme (tissue transglutaminase) that modulates fibrin crosslinking to form stable blood clots, making it a potential biomarker for cross-linked thrombi. Through ${ }^{99 \mathrm{~m}} \mathrm{Tc}$ radiolabeling, the peptide-based tracer ${ }^{99 \mathrm{~m}} \mathrm{Tc}$ NC100668 revealed specific detection of active factor (FXIII) signals in the lesions of a coronary microvascular disease mouse model. The relative retention of ${ }^{99 \mathrm{~m}} \mathrm{Tc}-\mathrm{NC} 100668$ (microvascular disease-to-septal region ratio) determined at $2 \mathrm{~h}$ was approximately 3 - to 12-fold higher than those acquired from 3 to $14 \mathrm{~d}$ after microvascular disease, suggesting its potential for the early detection 
of coronary microvascular disease associated with thrombus (37). Fibrin is typically upregulated in fresh thrombi and gradually replaced by collagen and other fibrotic protein, making the detection of fibrin an attractive strategy for identification of thrombosis and fibrosis. A ${ }^{64} \mathrm{Cu}$-radiolabeled fibrin binding probe 8 demonstrated favorable thrombus uptake, background clearance, and imaging efficacy in preclinical models and has been translated for human imaging (36).

\section{UNDEREXPLORED MOLECULAR IMAGING OF ADAPTIVE IMMUNE SYSTEM IN CVDS}

In addition to the innate immune system, the adaptive immune system also plays critical roles in tissue repair processes and fibrosis $(2,38)$. The pivotal role of T cells modulating cardiac fibroblasts and of MMP activity has been demonstrated in CVDs including heart failure, myocardial fibrosis, ischemia, and MI (39). The recent popularity of cancer immunotherapy has prompted the development of a range of T-cell imaging probes (40), which could be used to image subset $T$ cells to investigate the underlying mechanisms of tissue repair and fibrosis.

\section{CONCLUSIONS AND FUTURE DIRECTIONS}

Tissue repair and fibrosis are governed by the immune system. Balance between inflammatory and preparative immune responses guides the optimal tissue repair process. Thus, the immune-fibrosis axis is an unquestionable target for molecular imaging and immunomodulatory therapy. PET and SPECT imaging have shown great promise for visualizing signatures of the immune system, allowing insight into whether injured tissue will be properly repaired or subject to subsequent pathologic fibrosis. To date, a variety of radiotracers have been developed to detect the immune response and fibrosis in CVDs. Additional research needs to focus on the sensitivity and specificity of these radiotracers detecting the subtype of immune cells and fibroblasts. Moreover, longitudinal studies are required to uncover the connection between measured immune system activity and resulting fibrosis. Through the combination of multiple imaging agents targeting a range of biomarkers upregulated during the immune-fibrosis network, these studies will provide quantitative measurement of early-onset immune response, fibroblast activity, and subsequent pathologic fibrosis to elucidate the mechanism of crosstalk between the 2 systems and highlight the predictive value of molecular imaging. The early, sensitive, and specific detection of malfunctioning pathways causing pathologic fibrosis within the crosstalk network will enable the identification of potential therapeutic targets and provide real-time guidance to antifibrotic or targeted immunomodulatory therapy. The multimodality imaging using PET/MRI has great potential to differentiate subtypes of fibrosis (e.g., replacement vs. reactive fibrosis) and provide information on cellular and molecular profiles in those fibrotic lesions for better management. After establishment of a pathway for radiotracer translation for a first-in-humans study (22), these imaging strategies may hold the potential to decipher the heterogeneity of fibrotic diseases in patients for individualized treatment.

Taking together all these considerations, we envision a critical role for molecular imaging within the immune-fibrosis network to delineate the functions and interaction of immune cells and fibroblasts along the pathogenesis of fibrotic processes and to better elucidate the mechanisms of CVDs. The information we gather from ongoing clinical studies or future translational research will not only facilitate the development of diagnostic agents to phenotype and risk-stratify patients but also promote the discovery of novel therapeutic agents for targeted treatment.

\section{DISCLOSURE}

This work is supported by 1R35HL145212-01 from NHLBI. No other potential conflict of interest relevant to this article was reported.

\section{REFERENCES}

1. Wynn TA, Vannella KM. Macrophages in tissue repair, regeneration, and fibrosis. Immunity. 2016;44:450-462.

2. Gieseck RL III, Wilson MS, Wynn TA. Type 2 immunity in tissue repair and fibrosis. Nat Rev Immunol. 2018;18:62-76.

3. Frangogiannis NG. Cardiac fibrosis: cell biological mechanisms, molecular pathways and therapeutic opportunities. Mol Aspects Med. 2019;65:70-99.

4. Desogere P, Montesi SB, Caravan P. Molecular probes for imaging fibrosis and fibrogenesis. Chemistry. 2019;25:1128-1141.

5. Montesi SB, Desogere P, Fuchs BC, Caravan P. Molecular imaging of fibrosis: recent advances and future directions. J Clin Invest. 2019;129:24-33.

6. Heo GS, Sultan D, Liu Y. Current and novel radiopharmaceuticals for imaging cardiovascular inflammation. Q J Nucl Med Mol Imaging. 2020;64:4-20.

7. Siebermair J, Kohler MI, Kupusovic J, et al. Cardiac fibroblast activation detected by Ga-68 FAPI PET imaging as a potential novel biomarker of cardiac injury/ remodeling. J Nucl Cardiol. 2021;28:812-821.

8. Hess A, Thackeray JT, Wollert KC, Bengel FM. Radionuclide image-guided repair of the heart. JACC Cardiovasc Imaging. 2020;13:2415-2429.

9. Curley D, Lavin Plaza B, Shah AM, Botnar RM. Molecular imaging of cardiac remodelling after myocardial infarction. Basic Res Cardiol. 2018;113:10.

10. Eming SA, Wynn TA, Martin P. Inflammation and metabolism in tissue repair and regeneration. Science. 2017;356:1026-1030.

11. de Haas HJ, Arbustini E, Fuster V, Kramer CM, Narula J. Molecular imaging of the cardiac extracellular matrix. Circ Res. 2014;114:903-915.

12. Swirski FK, Nahrendorf M. Cardioimmunology: the immune system in cardiac homeostasis and disease. Nat Rev Immunol. 2018;18:733-744.

13. Hitscherich P, Lee EJ. Crosstalk between cardiac cells and macrophages postmyocardial infarction: insights from in vitro studies. Tissue Eng Part B Rev. December 3, 2020 [Epub ahead of print].

14. Sager HB, Kessler T, Schunkert H. Monocytes and macrophages in cardiac injury and repair. J Thorac Dis. 2017;9(suppl):S30-S35.

15. Thackeray JT, Bengel FM. Molecular imaging of myocardial inflammation with positron emission tomography post-ischemia: a determinant of subsequent remodeling or recovery. JACC Cardiovasc Imaging. 2018;11:1340-1355.

16. Hess A, Derlin T, Koenig T, et al. Molecular imaging-guided repair after acute myocardial infarction by targeting the chemokine receptor CXCR4. Eur Heart J. 2020;41:3564-3575.

17. Bajpai G, Bredemeyer A, Li W, et al. Tissue resident CCR2 - and CCR2 + cardiac macrophages differentially orchestrate monocyte recruitment and fate specification following myocardial injury. Circ Res. 2019;124:263-278.

18. Leuschner F, Courties G, Dutta P, et al. Silencing of CCR2 in myocarditis. Eur Heart J. 2015;36:1478-1488.

19. Heo GS, Kopecky B, Sultan D, et al. Molecular imaging visualizes recruitment of inflammatory monocytes and macrophages to the injured heart. Circ Res. 2019;124: 881-890.

20. Heo GS, Bajpai G, Li W, et al. Targeted PET imaging of chemokine receptor 2-positive monocytes and macrophages in the injured heart. $J$ Nucl Med. 2021;62: $111-114$.

21. Li W, Luehmann HP, Hsiao HM, et al. Visualization of monocytic cells in regressing atherosclerotic plaques by intravital two-photon and positron emission tomographybased imaging. Arterioscler Thromb Vasc Biol. 2018;38:1030-1036.

22. Brody SL, Gunsten SP, Luehmann HP, et al. Chemokine receptor 2-targeted molecular imaging in pulmonary fibrosis: a clinical trial. Am J Respir Crit Care Med. 2021;203:78-89.

23. 7 Studies found for: CCR2 PET. ClinicalTrials.gov website. https://www. clinicaltrials.gov/ct2/

results? cond $=\&$ term $=\mathrm{CCR} 2+\mathrm{PET} \&$ cntry $=\&$ state $=\&$ city $=\&$ dist $=$. Accessed August 9, 2021.

24. Wagner S, de Moura Gatti F, Silva DG, et al. Development of the first potential nonpeptidic positron emission tomography tracer for the imaging of CCR 2 receptors. ChemMedChem. 2021;16:640-645. 
25. DeLeon-Pennell KY, Meschiari CA, Jung M, Lindsey ML. Chapter two: matrix metalloproteinases in myocardial infarction and heart failure. In: Khalil RA, ed. Progress in Molecular Biology and Translational Science. Vol 147: Academic Press; 2017:75-100.

26. Toczek J, Ye Y, Gona K, et al. Preclinical evaluation of RYM1, a matrix metalloproteinase-targeted tracer for imaging aneurysm. J Nucl Med. 2017;58:1318-1323.

27. Li X, Rosenkrans ZT, Wang J, Cai W. PET imaging of macrophages in cardiovascular diseases. Am J Transl Res. 2020;12:1491-1514.

28. Gibb AA, Lazaropoulos MP, Elrod JW. Myofibroblasts and fibrosis: mitochondrial and metabolic control of cellular differentiation. Circ Res. 2020;127:427-447.

29. Altmann A, Haberkorn U, Siveke J. The latest developments in imaging of fibroblast activation protein. $J$ Nucl Med. 2021;62:160-167.

30. Aghajanian H, Kimura T, Rurik JG, et al. Targeting cardiac fibrosis with engineered T cells. Nature. 2019;573:430-433.

31. Varasteh Z, Mohanta S, Robu S, et al. Molecular imaging of fibroblast activity after myocardial infarction using a ${ }^{68} \mathrm{Ga}$-labeled fibroblast activation protein inhibitor, FAPI-04. J Nucl Med. 2019;60:1743-1749.

32. Heckmann MB, Reinhardt F, Finke D, et al. Relationship between cardiac fibroblast activation protein activity by positron emission tomography and cardiovascular disease. Circ Cardiovasc Imaging. 2020;13:e010628.
33. Eisinger F, Patzelt J, Langer HF. The platelet response to tissue injury. Front Med (Lausanne). 2018;5:317.

34. Ziegler M, Wang X, Peter K. Platelets in cardiac ischaemia/reperfusion injury: a promising therapeutic target. Cardiovasc Res. 2019;115:1178-1188.

35. Ziegler M, Alt K, Paterson BM, et al. Highly sensitive detection of minimal cardiac ischemia using positron emission tomography imaging of activated platelets. Sci Rep. 2016;6:38161.

36. Oliveira BL, Caravan P. Peptide-based fibrin-targeting probes for thrombus imaging. Dalton Trans. 2017;46:14488-14508.

37. Zhuang ZW, Huang Y, Ju R, et al. Molecular imaging of factor XIII activity for the early detection of mouse coronary microvascular disease. Theranostics. 2019;9: 1474-1489.

38. Sánchez-Trujillo L, Vazquez-Garza E, Castillo EC, Garcia-Rivas G, Torre-Amione G. Role of adaptive immunity in the development and progression of heart failure: new evidence. Arch Med Res. 2017;48:1-11.

39. Zhang M, Zhang S. T cells in fibrosis and fibrotic diseases. Front Immunol. 2020;11: 1142 .

40. Wei W, Jiang D, Ehlerding EB, Luo Q, Cai W. Noninvasive PET imaging of T cells. Trends Cancer. 2018;4:359-373. 